\title{
Progress and Persistent Challenges in Improving Care for Low- Income Women with Breast Cancer
}

\author{
Adrienne N. Cobb, MD, Taiwo Adesoye, MD MPH, and Mediget Teshome, MD MPH \\ Department of Breast Surgical Oncology, The University of Texas MD Anderson Cancer Center, Houston, TX \\ "We have also come to his hallowed spot to remind America of the fierce urgency of now. This is no time to engage in the luxury of cooling off or to take \\ the tranquilizing drug of gradualism." \\ Martin Luther King Jr, August 28, $1963^{1}$
}

Despite transformational advances in breast cancer management over the past several decades, disparities by race, ethnicity, and socioeconomic position persist across the care continuum from screening, diagnosis, treatment, and into survivorship. ${ }^{2-4}$ While multifactorial, in the US this phenomenon is largely rooted in societal inequities that intersect within a broader health care framework and are partially dependent upon access to care and health insurance status. $^{5,6}$ In 2010, the Patient Protection and Affordable Care Act (ACA) was adopted to improve health care access and expand insurance coverage in the US. This legislation included expanding Medicaid insurance to individuals below $138 \%$ of the federal poverty level as a core provision. ${ }^{7}$ While several studies have investigated the impact of the ACA on health outcomes, its influence on breast cancer health disparities has not been comprehensively defined.

In this issue of Annals of Surgical Oncology, ObengGyasi et al. ${ }^{8}$ report on the impact of Medicaid expansion in Ohio on surgical disparities affecting low-income breast cancer patients. Using data from the Ohio Cancer Incidence Surveillance System (a state registry with mandatory reporting) coupled with US Census information, the authors explore if Medicaid expansion mitigated existing disparities within three domains of breast cancer care: (1) the receipt of breast-conservation therapy (BCT), defined as breast-conserving surgery followed by adjuvant radiation (a surrogate for guideline-concordant local regional

(C) Society of Surgical Oncology 2022

First Received: 22 December 2021

Accepted: 10 January 2022;

Published Online: 12 February 2022

M. Teshome, MD MPH

e-mail: mteshome@mdanderson.org care); (2) the utilization of postmastectomy breast reconstruction; and (3) the time from diagnosis to surgery (days). The study cohort was compromised of women with nonmetastatic invasive breast cancer, diagnosed at age 30-64 years and treated with upfront surgery. Patients were further categorized by insurance status at diagnosis as either Medicaid/uninsured or privately insured. The authors defined the study period as pre-ACA (2010-2013) and post-ACA (2014-2017), punctuated by when Medicaid expansion was first implemented in Ohio (2014). The first 6 months after implementation represented a washout period and were not included in the analysis.

Over 16,000 patients were included in the analysis, with $14.5 \%$ of the total population uninsured or receiving Medicaid insurance. The authors report a significant reduction in the proportion of uninsured breast cancer patients in Ohio from $32.9 \%$ pre-ACA to $14.4 \%$ post-ACA. Medicaid expansion was associated with a significant increase in patients receiving BCT and postmastectomy breast reconstruction in both cohorts, with a higher magnitude noted in the Medicaid/uninsured group. The primary comparisons were statistically significant when evaluating pre-and post-ACA within the patient groups and via regression models comparing the Medicaid/uninsured and privately insured populations. Although not explicitly reported in absolute proportions as are derived here, the data presented illuminated an observed narrowing of the gap between breast cancer patients by insurance status from $9.7 \%$ pre-ACA to $5.1 \%$ post-ACA for the receipt of BCT and $15.6 \%$ pre-ACA to $9.6 \%$ post-ACA for the receipt of postmastectomy breast reconstruction. There was no change in the mean time to surgery or the proportion of patients who received surgery after 60 days of diagnosis in the Medicaid/uninsured group. 
Interestingly, among the privately insured group, a modest but statistically significant increase in mean time to surgery and proportion of patients who received surgery after 60 days of diagnosis was noted following Medicaid expansion. The etiology for this increase is not apparent from the study findings, however this may be related to a variety of factors, including increased utilization of preoperative services, transfer of care to another facility, second opinion, or other related factors. Despite this, as the authors astutely emphasize, the disparity in time to surgery persisted, with the Medicaid/uninsured patient group having a consistently longer time to surgery than those who were privately insured. A similar phenomenon has been described in other states such as Kentucky where a $40 \%$ increase in time from diagnosis to definitive surgery after Medicaid expansion was observed. ${ }^{9}$ This suggests that while expanding access may have improved the number of patients receiving treatment, the capacity of the health care system may not have grown in a compensatory fashion, thus ultimately disproportionately impacting low-income women who are more likely to receive care in a resourcepoor system. ${ }^{10}$

Timely receipt of breast surgery or definitive oncologic therapy has been linked to survival outcomes, further highlighting the acute need to address this worrisome finding. ${ }^{11}$ In breast cancer where multidisciplinary management may contribute to the complexity of care, challenges coordinating care prior to surgery may be one of the factors responsible for preoperative delays. ${ }^{12}$ In practical terms, appropriate timing of appointments and treatment planning, which may involve advanced breast imaging, additional biopsies, and multiple consultations with the multidisciplinary team, take time and are often performed on successive dates. This frequently requires travel to appointments and missed time from work, which can further compound the treatment timeline. As breast oncology care becomes more interconnected, health care delivery strategies to streamline evaluation and create efficient mechanisms for treatment planning become increasingly important to achieving optimal oncologic outcomes. Such strategies may include optimizing patient navigation through the healthcare system, use of patient advocates, accessibility to coordinated appointments, and greater ease of communication with physicians and ancillary staff.

Furthermore, several studies have described the secondary and often debilitating financial toxicity associated with oncology care. ${ }^{13-16}$ While patients in lower socioeconomic positions are clearly impacted by the significant direct and indirect treatment-related cost of care, patients who are insured also report high distress and negative financial impact from breast cancer treatment. ${ }^{17,18}$ With improving oncologic outcomes and a growing population of breast cancer survivors, greater attention to improving quality of life related to cost of care are paramount. ${ }^{19,20}$

Policy-based solutions addressing large structural issues such as cancer disparities within the health care system are urgently needed. Since its adoption in 2010, the ACA has greatly increased the number of insured Americans, leading to success, noted by the authors, in increased adoption of BCT and postmastectomy reconstruction. This legislation also eliminated cost-sharing for preventive services, including screening mammography, genetic counseling and testing for BRCA mutations, support for smoking cessation, and other important aspects focused on oncology care. ${ }^{21}$ Medicaid expansion as a mechanism to increase health care access has been variably implemented on a state-by state basis. Since its implementation, many investigators have explored the impact of Medicaid expansion on cancer care and oncologic outcomes describing diagnosis of breast cancer at earlier stages, ${ }^{9,22,23}$ increasing rates of breastconserving surgery, ${ }^{9}$ receipt of radiation ${ }^{23}$ and the utilization of breast reconstruction. ${ }^{24}$ Similarly, supported by this study, Medicaid expansion may also have the ability to partially accelerate and reduce the gap in observed and long-standing disparities in breast cancer treatment.

This study is limited by its retrospective nature and paucity of breast cancer-specific information such as tumor subtype, nodal stage, and treatment received. Stage was reported however, as a surrogate measure, defined broadly as either local (in breast disease only) regional (nodal involvement). Additionally, the relatively modest proportion of patients in the uninsured/Medicaid group may have impacted the research findings. Furthermore, insurance status is not static and may have changed over the years with patients moving between groups. Moreover, in defining the patient population, the authors excluded patients who were treated with neoadjuvant therapy, although this may be the preferred treatment strategy in certain cases, depending on tumor subtype and disease stage at diagnosis. Shared decision making remains a cornerstone of breast cancer treatment, with patient preference and factors such as family history, genetic predisposition, and inability to receive radiation impacting the treatment decision for breast-conserving surgery or mastectomy, and it is unclear to what extent this played a role in this study. Additionally, while receipt of breast cancer reconstruction improves with increasing access, some patients may not desire reconstruction or may not be candidates for immediate breast reconstruction.

Despite these limitations, this study provides an informative description of the impact of Medicaid expansion on surgical care for breast cancer in Ohio. The authors should be commended for this thoughtful investigation, which highlights a reduction in the gap between those with private 
insurance and those receiving Medicaid or uninsured in the receipt of BCT and postmastectomy reconstruction, although there was no improvement in reducing the disparity of time to surgery. This emphasizes the inadequacy of relying solely on access to insurance as a solution to health care disparities, suggesting that a multifaceted approach is essential. Furthermore, since Medicaid expansion is state-specific, it is highly relevant for participating and non-participating states alike to evaluate the role of insurance expansion (or non-expansion) in influencing health outcomes and care patterns among patients within this vulnerable socioeconomic position. Furthermore, the coronavirus disease 2019 (COVID-19) pandemic has undoubtedly strained the healthcare system and threatens to further widen existing disparities. Critical analysis, as demonstrated by Obeng-Gyasi and colleagues, may help define opportunities for deliberate refinement and innovative approaches, through national health policy and within health care institutions, to narrow, counteract, and ultimately eliminate these socioeconomic barriers.

The sobering reality that women with breast cancer in financially vulnerable populations are more susceptible to poor oncologic outcomes and worse survival represents a societal crisis. Although important improvements have been achieved in facilitating health care access, considerable work is nonetheless needed to ensure the timeliness of curative breast cancer treatment. We cannot accept poverty as a significant determinant of breast cancer outcomes and must aggressively, fiercely, and urgently pursue effective, targeted, and transformational strategies to achieve our shared goal of equitable breast cancer care.

\section{REFERENCES}

1. King ML, Jr. "I have a dream". USConstitution.net. August 28, 1963. Available at: https://www.usconstitution.net/dream.html. Accessed 12 Dec 2021.

2. Singh GK, Jemal A. Socioeconomic and racial/ethnic disparities in cancer mortality, incidence, and survival in the United States, 1950-2014: over six decades of changing patterns and widening inequalities. J Environ Public Health. 2017;2017:2819372.

3. DeSantis CE, Siegel RL, Sauer AG, et al. Cancer statistics for African Americans, 2016: progress and opportunities in reducing racial disparities. CA Cancer J Clin. 2016;66:290-308.

4. CancerDisparitiesProgressReport.org. Philadelphia, PA: American Association for Cancer Research; C2020 [16 Dec 2021]. Available at: http://www.CancerDisparitiesProgressReport.org/.

5. Williams DR, Mohammed SA, Shields AE. Understanding and effectively addressing breast cancer in African American women: unpacking the social context. Cancer. 2016;122:2138-49.

6. Newman LA, Kaljee LM. Health disparities and triple-negative breast cancer in African American women: a review. JAMA Surg. 2017;152:485-93.

7. Rosenbaum S, Westmoreland TM. The Supreme Court's surprising decision on the Medicaid expansion: how will the federal government and states proceed? Health Aff (Millwood). 2012;31:1663-72.
8. Obeng-Gyasi S, Rose J, Dong W, Kim U, Koroukian S. Is medicaid expansion narrowing gaps in surgical disparities for low-income breast cancer patients? Ann Surg Oncol. 2021. http s://doi.org/10.1245/s10434-021-11137-0.

9. Ajkay N, Bhutiani N, Huang B, et al. Early impact of Medicaid expansion and quality of breast cancer care in Kentucky. $J$ Am Coll Surg. 2018;226:498-504.

10. Semprini J, Olopade O. Evaluating the effect of Medicaid expansion on black/white breast cancer mortality disparities: a difference-in-difference analysis. JCO Glob Oncol. 2020;6:1178-83.

11. Bleicher RJ, Ruth K, Sigurdson ER, et al. Time to surgery and breast cancer survival in the United States. JAMA Oncol. 2016;2:330-9.

12. Bleicher RJ, Ruth K, Sigurdson ER, et al. Preoperative delays in the US Medicare population with breast cancer. J Clin Oncol. 2012;30:4485-92.

13. Greenup RA, Rushing C, Fish L, et al. Financial costs and burden related to decisions for breast cancer surgery. J Oncol Pract. 2019;15:e666-76.

14. Ramsey S, Blough D, Kirchhoff A, et al. Washington State cancer patients found to be at greater risk for bankruptcy than people without a cancer diagnosis. Health Aff (Millwood). 2013;32:1143-52.

15. Tucker-Seeley RD, Yabroff KR. Minimizing the "financial toxicity" associated with cancer care: advancing the research agenda. J Natl Cancer Inst. 2015;108(5):djv410.

16. Boukovalas S, Liu J, Asaad M, et al. Relationship between financial toxicity and surgical treatment for early-stage breast cancer: a propensity score-matched comparison of breast-conserving therapy and mastectomy. $J$ Am Coll Surg. 2021;233:445.e2-56.e2.

17. Offodile AC 2nd, Asaad M, Boukovalas S, et al. Financial toxicity following surgical treatment for breast cancer: a crosssectional pilot study. Ann Surg Oncol. 2021;28:2451-62.

18. Gilligan AM, Alberts DS, Roe DJ, Skrepnek GH. Death or debt? National estimates of financial toxicity in persons with newlydiagnosed cancer. Am J Med. 2018;131:1187.e5-99.e5.

19. Zafar SY, McNeil RB, Thomas CM, Lathan CS, Ayanian JZ, Provenzale D. Population-based assessment of cancer survivors' financial burden and quality of life: a prospective cohort study. $J$ Oncol Pract. 2014;11:145-50.

20. Lentz R, Benson Iii AB, Kircher S. Financial toxicity in cancer care: prevalence, causes, consequences, and reduction strategies. J Surg Oncol. 2019;120:85-92.

21. Zhao J, Mao Z, Fedewa SA, et al. The Affordable Care Act and access to care across the cancer control continuum: a review at 10 years. CA Cancer J Clin. 2020;70:165-81.

22. Takvorian SU, Oganisian A, Mamtani R, et al. Association of Medicaid expansion under the Affordable Care Act with insurance status, cancer stage, and timely treatment among patients with breast, colon, and lung cancer. JAMA Netw Open. 2020;3:e1921653-e.

23. Chu QD, Li T, Hsieh MC, et al. Positive impact of the Patient Protection and Affordable Care Act Medicaid expansion on Louisiana women with breast cancer. Cancer. 2021;127:688-99.

24. Ramalingam K, Ji L, Pairawan S, Molina DC, Lum SS. Improvement in breast reconstruction disparities following Medicaid expansion under the Affordable Care Act. Ann Surg Oncol. 2021;28:5558-67.

Publisher's Note Springer Nature remains neutral with regard to jurisdictional claims in published maps and institutional affiliations. 